\title{
Noninfectious but Genetic Bilateral Neck Swelling
}

Capraro J (1), Nagel W (2), Clerici Th (2), Stöckli S (3), Brändle M (1), Bilz S (1)

(1) Division of Endocrinology and Diabetes (2) Division of Surgery and (3) Otolaryngology, Kantonsspital

St. Gallen, Switzerland

\section{Background:}

Head and neck paragangliomas (HNPGLs) are rare tumors mostly arising from the parasympathic paraganglia such as the carotid body. They are usually detected because of local mass effects or during the work-up of a genetic syndrome. We describe a case of a 32 year old woman with bilateral carotid body tumors.

\section{Case Report}

A 32-year old previously healthy mother of 3 children was referred because of bilateral neck swelling, presumably lymphadenopathy, associated with recurrent upper respiratory tract infections during the past year. Cervical ultrasound raised the suspicion for bilateral carotid body tumors which were confirmed by MR-imaging and a subsequent DOPA-PET-CT, which showed no evidence for multifocality or metastases. Plasma metanephrines including methoxy-tyramine were within the normal range and although a detailed family history revealed no evidence for a syndromic presention, the genetic work-up revealed a SDHD mutation and the diagnosis of paraganglioma syndrome typ 1 Because of impending mass effects and the prospect of a curative approach according to the result of an MR-angiography, resection of the tumors has been performed.
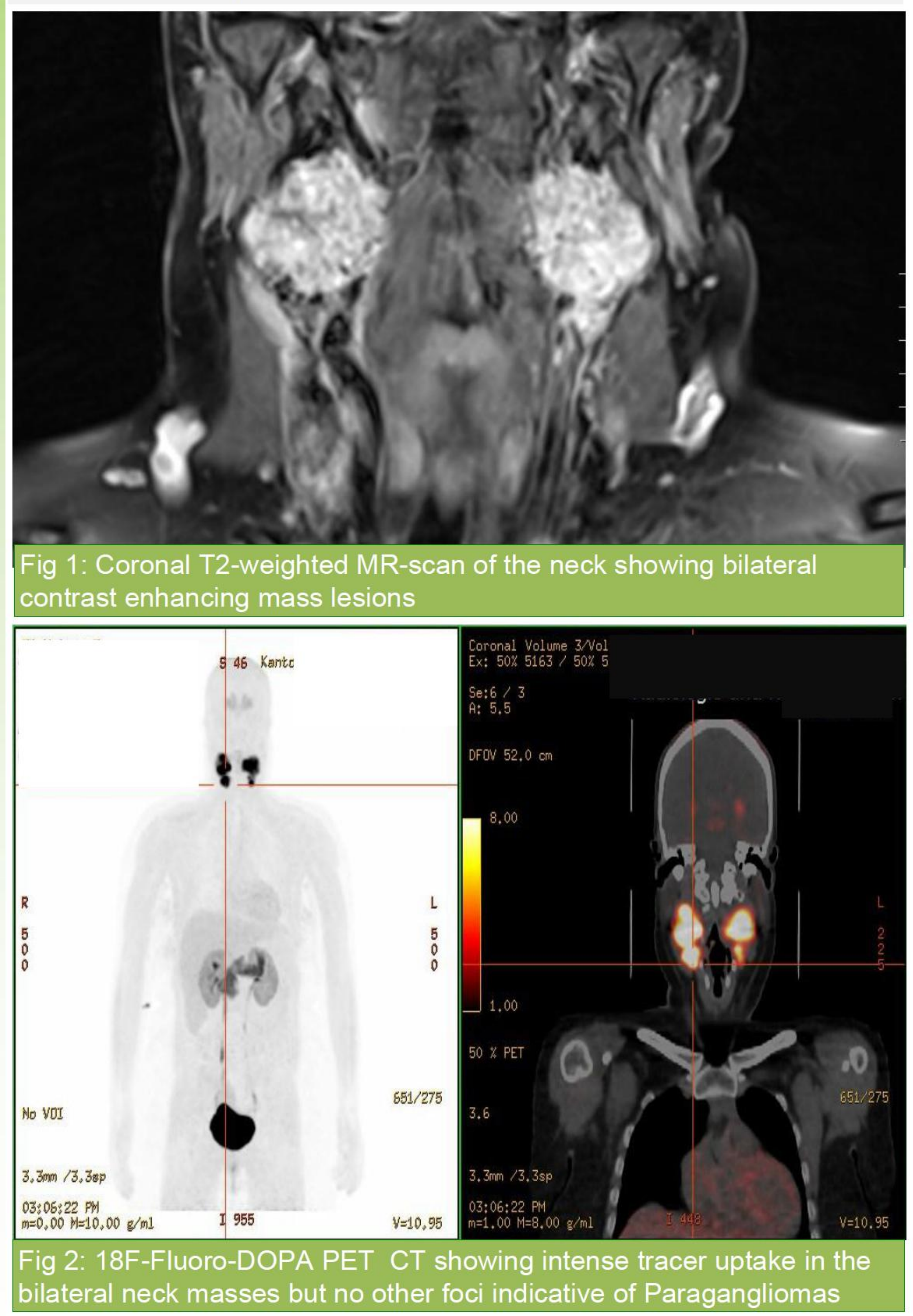

\section{References}

Lenders JW et al;; Pheochromocytoma and paraganglioma: an endocrine society clinical practice guideline, J Clin Endocrinol Metab. 2014 Jun;99(6):1915-42
Taieb D et a.:; Current Approaches and Recent Developments in the Management of Head and Neck Paragangliomas; Endocrine Reviews, October 2014, $35(5) 795-819$

\section{Discussion:}

HNPGLs are rare tumors (incidence 1:30'000 - 100'000) derived from extraadrenal chromaffin cells and $95 \%$ arise from the parasympathic nervous system, typically the carotid body (glomus caroticum), jugular bulb (glomus jugulare) and different branches of the vagal nerve (glomus vagale, glomus typanicum). Although plasma methoxytyramine may be elevated in up to one third, they are clinically nonsecretory in most cases and present because of their mass effects. Around 35\% are associated with a genetic defect, with SDHD-mutations accounting for > $50 \%$, SDHB for $20-35 \%$ and SDHC for $15 \%$. Mutations in other PHEO/PGL susceptibility genes such as SDHAF2 are exceptional. The inheritance pattern is autosomal dominant for SDHx mutations, but in SDHD and SDHAF2 the disease occurs only, if the mutation is inherited from the father, which is in line with maternal imprinting (=imprinted and not expressed in the offspring). Malignancy is rare (3.5\%) although metastases occur up to 20 years after initial diagnosis. For both diagnosis and detection of multifocality and/or malignancy functional imaging with $18 \mathrm{~F}-\mathrm{DOPA}$ - or $68 \mathrm{Ga}-\mathrm{DOTATATE}-\mathrm{PET}-\mathrm{CT}$ is considered superior whereas anatomical imaging with $\mathrm{CT}$ and/or MRI gives a better locoregional resolution. Surgical resection should be attempted in referral centers but may be associated with significant morbidity including cranial nerve injuries. The requirement for perioperative alphaadrenergic blockade is assessed by biochemical testing. Radiotherapy/surgery can be applied to non-resectable symptomatic tumors and chemotherapy is reserved for advanced/progressive disease. Genetic counselling and testing is mandatory due to the high rate of germline mutations and familial disease.

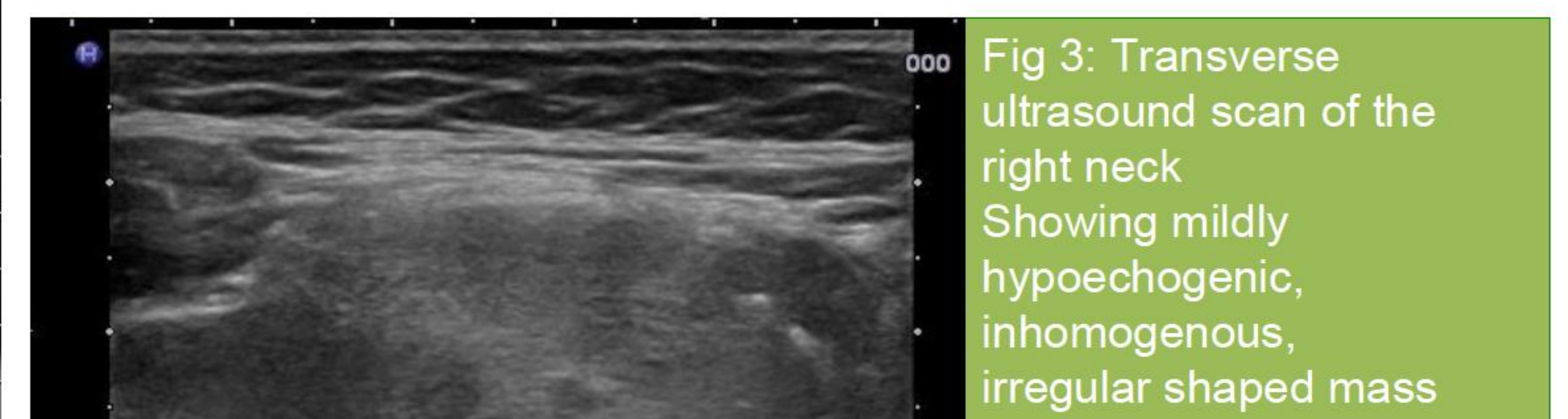

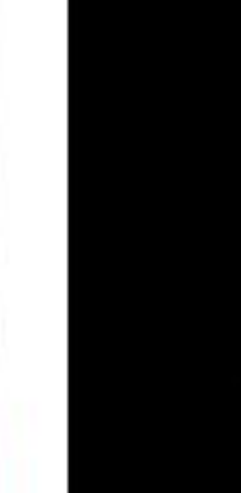
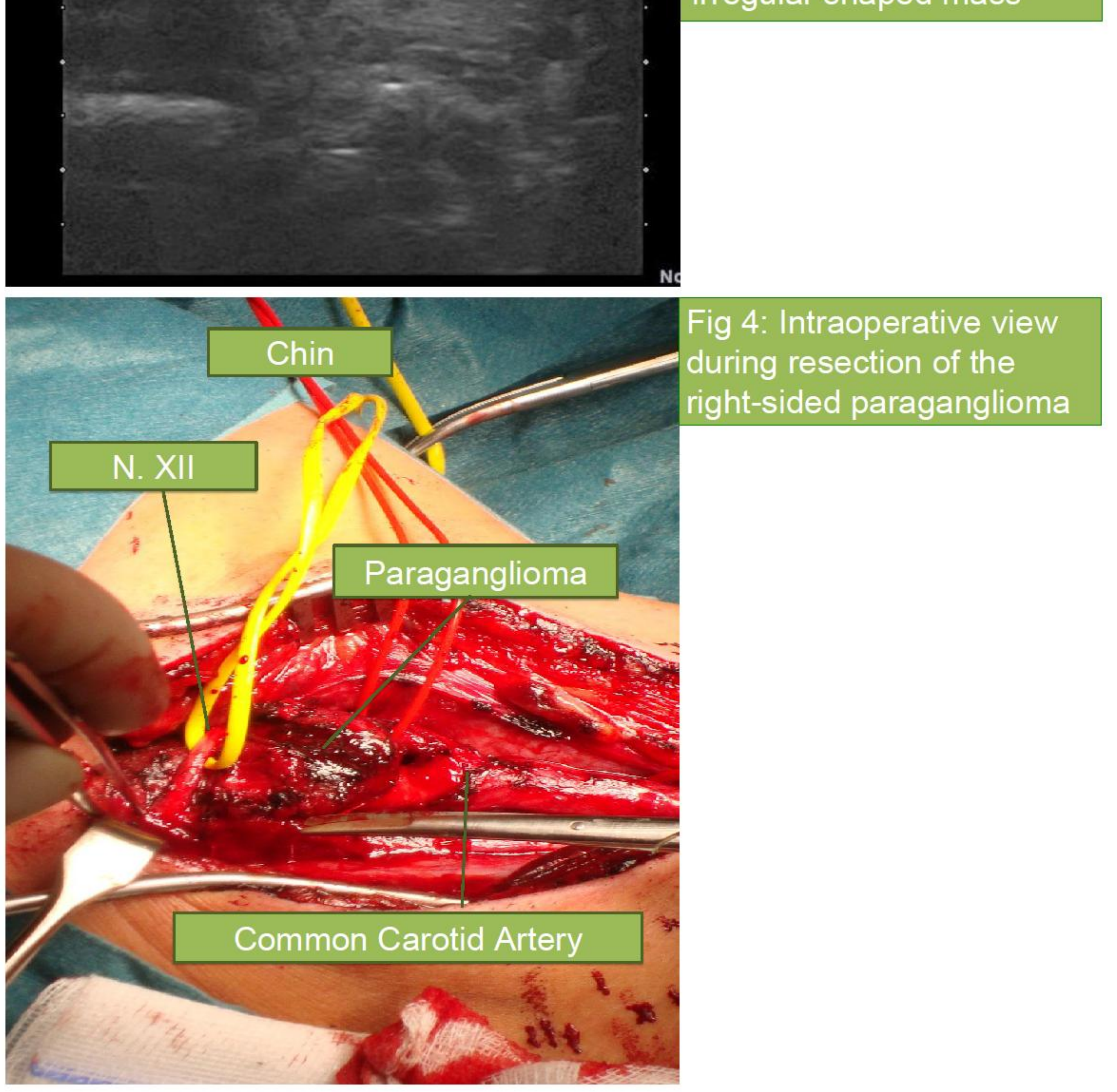